J. theor. Biol. (1972) 34, 451-467

\title{
Systems Approach to the Study of Drug Transport across Membranes using Suspension Cultures of Mammalian Cells
}

\section{Theoretical Diffusion Models}

\author{
N. F. H. Ho, J. Turi, C. Shipman, JR AND W. I. Higuchi \\ The College of Pharmacy
}

The Department of Microbiology and the Dental Research Institute

The University of Michigan, Ann Arbor, Mich. 48104, U.S.A.

(Received 15 July 1970, and in revised form 9 April 1971)

\begin{abstract}
Some general physical models are described for the diffusional transport of drugs across membranes of cells in culture suspensions. The models provide a basis for the design and analysis of experiments that are aimed to describe (a) the nature of the principal transport barrier, (b) the kinds of drug species being transported, (c) whether, where and how much solute binding occurs, and (d) the influences of $\mathrm{pH}$, partition coefficient and numerous other factors. The cell is treated as a sphere with non-homogeneous phase compartments. Both rigorous and approximate mathematical expressions have been derived for the quasi-steady-state diffusion through the membrane followed by three cases accounting for the distribution of drug in the heterogeneous cell interior, that is, (a) the non-steady-state situation, (b) establishment of instantaneous distribution and (c) instantaneous distribution in the aqueous interior with slow permeation of drug into the cytoplasmic bodies and nucleus.
\end{abstract}

\section{Introduction}

Suspension cultures of mammalian cells provide a unique system for drug transport studies. There are in vitro studies that utilize monolayers (Miller, 1968), lipid bilayers (Bangham, Standish \& Watkins, 1965; Sessa \& Weissmann, 1968; Bean, Shepperd \& Chan, 1968; Finkelstein \& Cass, 1968), interfacial films of oil/water emulsion droplets (Goldberg, Higuchi, Ho \& Zografi, 1967; Goldberg, 1968; Bikhazi, 1970) and gelatin-oil coacervates (Ghanem, Higuchi \& Simonelli, 1969, 1970) as model biological membranes. There are also numerous in situ investigations of drug transport across the gastric, intestinal, rectal, buccal and peritoneal and cutaneous walls of 
animals (Kakemi, Arita \& Muranishi, 1965; Kakemi, Sezaki, Muranishi \& Tsujimura, 1969; Wagner, 1968; Beckett \& Triggs, 1967; Beckett \& Moffat, 1968; Penzotti \& Mattocks, 1968; Mattocks, 1969; Elfbaum \& Laden, 1967, 1968; Scheuplein, Blank, Brauner \& MacFarlane, 1969). However, cell culture suspensions present membranes for transport studies and may uniquely provide the means to interrelate clinical and animal experiments on drug transport with physicochemical theories and model experiments. Although a cell culture suspension is an in vitro system and, therefore, subject to certain biological artifacts, intact cell lines in some state of metabolism place one closer to biological membranes with the distinct advantage of in vitro controls. For example, the Burkitt lymphoma cell system (Epstein, Achong \& Barr, 1964) is presently employed as a model for the molecular mechanistic interpretation of the influences of various factors upon the transport of drugs across cell wall membranes (Plate I).

Transport processes across membranes of bacterial and mammalian cells have become a topic of high interest. The amount of research is voluminous (Tuwiner, 1962; Christensen, 1962; Troshin, 1966; Stein, 1967). Likewise, descriptions of physicochemical models are abundant in the literature (Ling, 1962; Zwolinski, Eyring \& Reese, 1949; Lakshminarayanaiah, 1965; Curran $\&$ Schultz, 1968). Considerable emphasis has been placed on the transport of electrolytes, sugars, alcohols and amino acids into erythrocytes. The uptake and metabolism of cholesterol in tissue culture cells has been studied by Rothblat, Hartzell, Mialhe \& Kritchevsky (1966) and Rothblat, Buchko \& Kritchevsky (1968).

The purpose of this paper is to describe some rather general physical models for the diffusional transport of drugs across membranes of cells in culture suspensions. The models are attuned to the laboratory situation providing the basis for the design and analysis of experiments to describe (a) the nature of the principal transport barrier, (b) the kinds of drug species being transported, (c) whether, where and how much solute binding occurs, and (d) the influences of $\mathrm{pH}$, partition coefficient and numerous other factors.

\section{General Description of the Models}

The physical models are formulated for the passive diffusion of drug through multi-phase spherical compartments. Only unionized drug species are assumed to transfer into the lipid environment. Ionic equilibria are governed by the conditions in the aqueous phase and the properties of the drug, i.e. whether it is amphoteric, neutral, acidic or basic. The $\left[\mathrm{H}^{+}\right]$in any one compartment is assumed to be constant, which implies the existence of a high buffer capacity. No immediate considerations are given to the release 


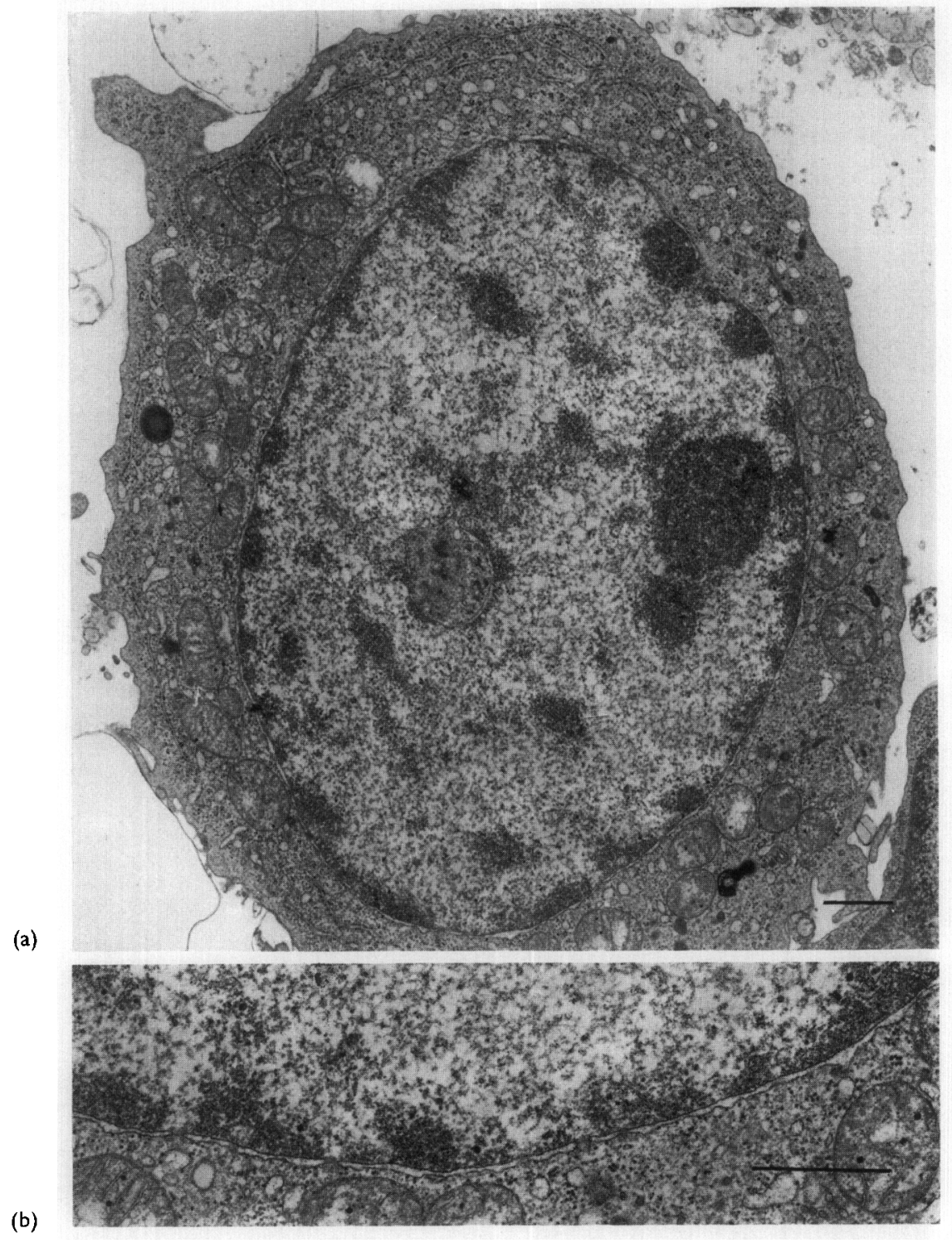

PLATE I. (a) Electron micrograph of a $\mathbf{P}_{3} \mathbf{J}$ Burkitt lymphoma cell. (b) An enlarged portion of the cell illustrating the nuclear membrane with pores. Each magnification marker represents $1 \mu \mathrm{m}$. 
of acidic products from the cell to the bulk solution nor to the effects of osmotic pressure, clectrical field and chemical reactions. Adsorption, however, is considered.

The boundary conditions for the uptake of drug in the bulk aqueous phase by the cells in suspension at zero time is

$$
\begin{array}{ll}
Y_{\mathrm{AQ}}=Y_{\mathrm{AQ}}(0), & r>a, \\
Y_{\text {cell }}=0 & r \leqslant a,
\end{array}
$$

where $Y_{\mathrm{AQ}}$ is the total drug concentration in the bulk aqueous phase, $Y_{\text {cell }}$ is the total concentration of drug within the cell taken as a sphere of radius $a$.

In the models it is assumed that there is a quasi-steady-state flux of drug across the plasma membrane, an assumption which is justified by the thinness of this layer. The membrane is assumed to consist of lipids and aqueous pores. As soon as the drug permeates through the plasma membrane and enters the cell interior, the drug distribution in the interior may follow one of three principal models.

Model 1. Non-steady-state distribution in the heterogeneous cell interior.

Model 2. Rapid (instantaneous) equilibration in the heterogeneous cell interior.

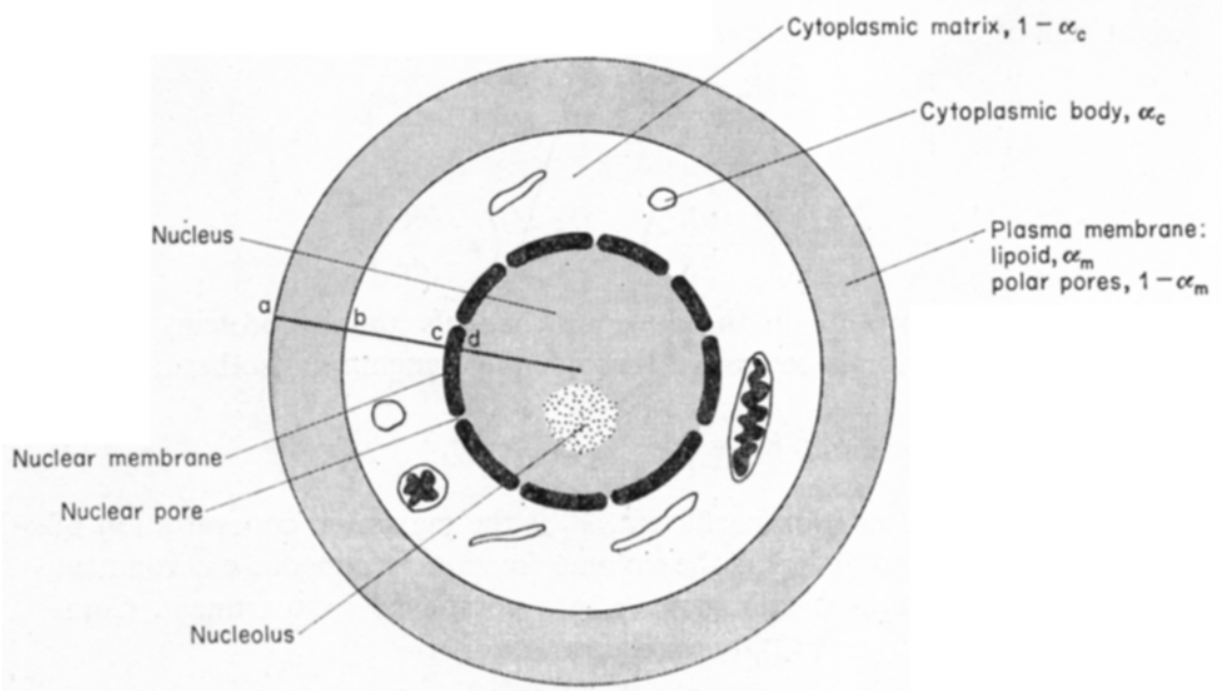

FIG. 1. Schematic description of the mammalian cell used for the development of the theoretical diffusional drug transport models. 
Model 3. Rapid (instantaneous) equilibration in aqueous environment with slow simultancous permeation of drug into the cytoplasmic bodies and nucleus.

A schematic diagram of the cell used for these models is shown in Fig. 1.

\section{General Rate of Change of Each Drug Species in an Aqueous Environment}

In the aqueous environment of a composite compartment, such as the aqueous channels of the plasma and nuclear membrane and the aqueous interior of the cell, some drug species are free to diffuse and others are adsorbed to the walls of the channel and by protein molecules. The rate of change of the concentration of any one kind of drug species can be described by Fick's second law for radial diffusion in a sphere. Similarly, after Liang \& Tong (1969),

$$
\frac{\partial\left(R_{\mathrm{w}}+R_{\mathrm{w}}^{*}\right)}{\partial t}=\frac{D_{\mathrm{w}}}{r^{2}} \frac{\partial}{\partial r}\left(r^{2} \frac{\partial R_{\mathrm{w}}}{\partial r}\right)+\frac{D_{\mathrm{w}}^{*}}{r^{2}} \frac{\partial}{\partial r}\left(r^{2} \frac{\partial R_{\mathrm{w}}^{*}}{\partial r}\right),
$$

where $R_{\mathrm{w}}$ is the concentration of neutral or ionic species in the aqueous solution, $R_{\mathrm{w}}^{*}$ is the concentration of adsorbed species, $D_{\mathrm{w}}$ and $D_{\mathrm{w}}^{*}$ are the aqueous diffusion coefficient of the free and the adsorbed drug species.

If the adsorbed species are immobile, i.e. $D_{\mathrm{w}}^{*} \sim 0$, the second term on the right side of the equation is zero. It follows that

$$
\frac{\partial\left(R_{\mathrm{w}}+R_{\mathrm{w}}^{*}\right)}{\partial t}=\frac{D_{\mathrm{w}}}{r^{2}} \frac{\partial}{\partial r}\left(r^{2} \frac{\partial R_{\mathrm{w}}}{\partial r}\right)
$$

or

$$
\frac{\partial R_{\mathrm{w}}}{\partial t}\left(1+\frac{\mathrm{d} R_{\mathrm{w}}^{*}}{\mathrm{~d} R_{\mathrm{w}}}\right)=\frac{D_{\mathrm{w}}}{r^{2}} \frac{\partial}{\partial r}\left(r^{2} \frac{\partial R_{\mathrm{w}}}{\partial r}\right) .
$$

Assume that adsorption in the aqueous channels and by proteins in the aqueous cell interior is reversible through the Langmuir's isotherm; thus,

$$
R_{\mathrm{w}}^{*}=\frac{k_{1} k_{2} R_{\mathrm{w}}}{1+k_{1} R_{\mathrm{w}}} \cdot(1-\alpha),
$$

where $k_{1}$ is the adsorption coefficient, $k_{2}$ is the maximum concentration of adsorption sites and $(1-\alpha)$ is the volume fraction of aqueous environment where the adsorption is taking place in a composite compartment. Combining equations (2) and (3), one obtains

$$
\frac{\partial R_{\mathrm{w}}}{\partial t}=\frac{D_{\mathrm{w}}}{1+(1-\alpha) k_{1} k_{2} /\left(1+k_{1} R_{\mathrm{w}}\right)^{2}} \cdot \frac{1}{r^{2}} \frac{\partial}{\partial r}\left(r^{2} \frac{\partial R_{\mathrm{w}}}{\partial r}\right)
$$


and the equation cannot be solved analytically. The term

$$
\frac{D_{\mathrm{w}}}{1+(1-\alpha) k_{1} k_{2} /\left(1+k_{1} R_{\mathrm{w}}\right)^{2}}
$$

makes the effective diffusion coefficient of the system concentrationdependent. When the amount adsorbed relative to the maximum amount that can be adsorbed is small, i.e. the region of linear adsorption, equation (4) becomes a linear differential equation,

$$
\frac{\partial R_{\mathrm{w}}}{\partial t}=\frac{D_{\mathrm{w}}}{1+(1-\alpha) k_{1} k_{2}} \cdot \frac{1}{r^{2}} \frac{\partial}{\partial r}\left(r^{2} \frac{\partial R_{\mathrm{w}}}{\partial r}\right) .
$$

At the quasi-steady-state, $\partial R_{\mathrm{w}} / \partial t \sim 0$ and

$$
G=\frac{4 \pi D_{\mathrm{w}} r^{2}}{E} \frac{\partial R_{\mathrm{w}}}{\partial r}, \quad(r \leqslant a),
$$

where $G$ is the inward quasi-steady-state flux of a drug species across a spherical surface and $E=1+(1-\alpha) k_{1} k_{2} \geqslant 1$. Here, perhaps contrary to custom, the inward flux is taken as positive in the direction of decreasing distances.

\section{General Description of a Drug and its Distribution in a Compartment}

In a previous paper (Suzuki, Higuchi \& Ho, 1970) a drug was described in the following manner.

$$
\begin{gathered}
\text { Basic drug: } \quad \mathrm{R}_{\mathrm{w}}^{+} \rightleftharpoons \mathrm{R}_{\mathrm{w}}^{\circ}+\mathrm{H}^{+} . \\
\text {Acidic drug: } \quad \mathrm{R}_{\mathrm{w}}^{\circ} \rightleftharpoons \mathrm{R}_{\mathrm{w}}^{-}+\mathrm{H}^{+} . \\
K_{1}=\left[\mathrm{R}_{\mathrm{w}}^{\circ}\right]\left[\mathrm{H}^{+}\right] /\left[\mathrm{R}_{\mathrm{w}}^{+}\right], \\
K_{2}=\left[\mathrm{R}_{\mathrm{w}}^{-}\right]\left[\mathrm{H}^{+}\right] /\left[\mathrm{R}_{\mathrm{w}}^{\circ}\right] .
\end{gathered}
$$

For a basic drug the dissociation constant is $K_{1}$ with $K_{2}=0$. For an acidic drug the dissociation constant is $K_{2}$ with $K_{1}=\infty$; and for a neutral drug, $K_{1}=\infty$ and $K_{2}=0$.

In general, the total concentration of drug as a function of position and time in any spherical compartment is

$$
Y(r, t)=\alpha\left[\mathrm{R}_{0}^{\circ}\right]+(1-\alpha)\left(\left[\mathrm{R}_{\mathrm{w}}^{+}\right]+\left[\mathrm{R}_{\mathrm{w}}^{\circ}\right]+\left[\mathrm{R}_{\mathrm{w}}^{-}\right]\right),
$$

where $Y(r, t)$ is the total drug concentration; $\alpha$ is the volume fraction of lipids or cytoplasmic bodies; $\left[\mathrm{R}_{\mathrm{w}}^{+}\right],\left[\mathrm{R}_{\mathrm{w}}^{\circ}\right]$ and $\left[\mathrm{R}_{\mathrm{w}}^{-}\right]$are the concentrations of cationic, neutral and anionic drug species; the subscripts 0 and $w$ represent the lipid and aqueous phases. It is assumed that only non-ionized drug 
species can partition into the lipid phase; thus, the partition coefficient $K$ is

$$
K=\left[\mathrm{R}_{0}^{\circ}\right] /\left[\mathrm{R}_{\mathrm{w}}^{\circ}\right] .
$$

After substituting equations (7), (8) and (10) into (9) and rearranging, we find that

where

$$
\begin{aligned}
& {\left[\mathrm{R}_{0}^{\circ}\right]=C_{0}^{\circ} Y(r, t),} \\
& {\left[\mathrm{R}_{\mathrm{w}}^{+}\right]=C_{\mathrm{w}}^{+} Y(r, t),} \\
& {\left[\mathrm{R}_{\mathrm{w}}^{\circ}\right]=C_{\mathrm{w}}^{\circ} Y(r, t),} \\
& {\left[\mathrm{R}_{\mathrm{w}}^{-}\right]=C_{\mathrm{w}}^{-} Y(r, t),}
\end{aligned}
$$

$$
\begin{aligned}
& C_{0}^{\circ}=K\left[\mathrm{H}^{+}\right] K_{1} / \beta, \\
& C_{\mathrm{w}}^{+}=\left[\mathrm{H}^{+}\right]^{2} / \beta, \\
& C_{\mathrm{w}}^{\circ}=\left[\mathrm{H}^{+}\right] K_{1} / \beta, \\
& C_{\mathrm{w}}^{-}=K_{1} K_{2} / \beta, \\
& \beta=\alpha K K_{1}\left[\mathrm{H}^{+}\right]+(1-\alpha)\left(\left[\mathrm{H}^{+}\right]^{2}+K_{1}\left[\mathrm{H}^{+}\right]+K_{1} K_{2}\right),
\end{aligned}
$$

where $C_{0}^{\circ}, C_{\mathrm{w}}^{+}$, etc. represent the fraction of non-ionized, cationic species, etc. in the lipid and water phases, respectively.

\section{Rate of Change of Drug Concentration in the Plasma Membrane}

The quasi-steady-state flux of all drug species in the heterogeneous membrane can be expressed by

$$
\begin{aligned}
G_{\mathrm{M}} & =4 \pi N r^{2} \frac{\partial \sum D \cdot[\mathrm{R}]}{\partial r} \\
& =-4 \pi N \frac{\partial \sum D \cdot[\mathrm{R}]}{\partial(1 / r)}, \quad(a \geqslant r \geqslant b),
\end{aligned}
$$

where $G_{\mathrm{M}}$ is the flux in the plasma membrane, $N$ is the number of cells and $\sum D \cdot[\mathrm{R}]$ is the sum of the product of the diffusion coefficient and the concentration of each respective drug species in the heterogeneous membrane. The thinness of the membrane permits the assumption of a linear concentration gradient across the region of diffusion. Utilizing equation (6) and taking the outer and inner radii of the cell to be $a$ and $b$ with $(a-b)$ being the thickness of the membrane, we expand equation (13) as follows:

$$
\begin{aligned}
G_{\mathrm{M}}= & \frac{4 \pi N a b}{(a-b)}\left\{\alpha_{\mathrm{M}} D_{0}^{\circ}\left(R_{0, a}^{\circ}-R_{0, b}^{\circ}\right)+\left(1-\alpha_{\mathrm{M}}\right)\right. \\
& {\left.\left[\frac{D_{\mathrm{w}}^{+}}{E_{\mathrm{M}}^{+}}\left(R_{\mathrm{w}, a}^{+}-R_{\mathrm{w}, b}^{+}\right)+\frac{D_{\mathrm{w}}^{\circ}}{E_{\mathrm{M}}^{\circ}}\left(R_{\mathrm{w}, a}^{\circ}-R_{\mathrm{w}, b}^{\circ}\right)+\frac{D_{\mathrm{w}}^{-}}{E_{\mathrm{M}}^{-}}\left(R_{\mathrm{w}, a}^{-}-R_{\mathrm{w}, b}^{-}\right)\right]\right\} . }
\end{aligned}
$$


Substituting equation (11) into (14),

where

$$
G_{\mathrm{M}}=\frac{4 \pi N a b}{(a-b)} D_{\mathrm{e}, \mathrm{M}}\left(Y_{\mathrm{M}, a}-Y_{\mathrm{M}, b}\right)
$$

$$
D_{\mathrm{e}, \mathrm{M}}=\alpha_{\mathrm{M}} D_{0}^{\circ} C_{0}^{\circ}+\left(1-\alpha_{\mathrm{M}}\right)\left[\frac{D_{\mathrm{w}}^{+}}{E_{\mathrm{M}}^{+}} C_{\mathrm{w}}^{+}+\frac{D_{\mathrm{w}}^{\circ}}{E_{\mathrm{M}}^{\circ}} C_{\mathrm{w}}^{\circ}+\frac{D_{\mathrm{w}}^{-}}{E_{\mathrm{M}}^{-}} C_{\mathrm{w}}^{-}\right] \text {. }
$$

Thus, the effective diffusion coefficient $\left(D_{\mathrm{e}, \mathrm{M}}\right)$ in the membrane simultaneously accounts for the intrinsic diffusivity of non-ionized drug species in the lipid phase and all species in the aqueous channels, including the modifying influence of linear equilibrium adsorption in the channels as described before.

The effective partition coefficients at $r=a$ and $b$ are

$$
\begin{aligned}
& K_{\mathrm{e}, a}=Y_{\mathrm{M}, a} / Y_{\mathrm{AQ}}, \\
& K_{\mathrm{e}, b}=Y_{\mathrm{IN}, b} / Y_{\mathrm{M}, b},
\end{aligned}
$$

where $Y_{\mathrm{AQ}}, Y_{\mathrm{M}, a}$ and $Y_{\mathrm{IN}, b}$ are the total drug concentrations in the bulk aqueous phase, membrane at $r=a$ and interior of the cell at $r=b$, respectively. It is assumed that the equilibrium is established instantaneously at the interface. It follows immediately that equation (15) becomes

$$
G_{\mathrm{M}}=\frac{4 \pi N a b D_{\mathrm{e}, \mathrm{M}}}{K_{\mathrm{e}, b}(a-b)}\left(K_{\mathrm{e}, a} K_{\mathrm{e}, b} Y_{\mathrm{AQ}}-Y_{\mathrm{IN}, b}\right),
$$

whereupon the effective permeability coefficient $\left(P_{\mathrm{e}, \mathrm{M}}\right)$ of the membrane is a constant and is defined as

$$
P_{\mathrm{e}, \mathrm{M}}=D_{\mathrm{e}, \mathrm{M}} K_{\mathrm{e}, a} /(a-b) .
$$

The total rate of change of drug in the bulk aqueous phase is related to the total flux in the membrane; then

$$
G_{\mathrm{M}}=-V_{\mathrm{AQ}} \frac{\mathrm{d} Y_{\mathrm{AQ}}}{\mathrm{d} t}
$$

where $V_{\mathrm{AQ}}$ is the volume of the bulk aqueous phase. Accordingly,

$$
-\frac{\mathrm{d} Y_{\mathrm{AQ}}}{\mathrm{d} t}=\frac{4 \pi N a b P_{\mathrm{e}, \mathrm{M}}}{V_{\mathrm{AQ}}}\left(Y_{\mathrm{AQ}}-\frac{Y_{\mathrm{IN}, b}}{K_{\mathrm{e}, a} K_{\mathrm{e}, b}}\right) .
$$

When $\mathrm{d} Y_{\mathrm{AQ}} / \mathrm{d} t=0$ at $t=\infty$,

$$
K_{e, a} K_{e, b}=Y_{\mathrm{IN}(\infty)} / Y_{\mathrm{AQ}(\infty)} .
$$

Equation (22) is the primary equation for the steady-state flux of drug in the membrane. Now it is necessary to describe the distribution of drug in the cell interior with time according to the three models previously indicated. 
In this and other diffusion models to follow the membrane has been treated as a compartment in which the passage of drug molecules occurs by diffusion through real or statistical aqueous pores and by partitioning and solubilization in the lipoidal membrane. It is well known that transport of solutes across membranes cannot often be explained by diffusion alone. Thus, the detailed description of the effective permeability coefficient, $P_{e, M}$, in these present models is admittedly a limiting one. Nevertheless, there will exist a $P_{e, M}$ so that equation (22) is generally applicable.

\section{Rate of Drug Transport into Cells using Model 1}

This model assumes non-steady-state diffusion within the cell composed of cytoplasmic bodies and the nucleus in an aqueous phase.

$$
\frac{\partial Y_{\mathrm{IN}}}{\partial t}=\frac{D_{\varepsilon, \mathrm{IN}}}{r^{2}} \frac{\partial}{\partial r}\left(r^{2} \frac{\partial Y_{\mathrm{IN}}}{\partial r}\right) \quad(0<r<b)
$$

where

$$
D_{\mathrm{e}, \mathrm{IN}}=\alpha_{\mathrm{IN}} D_{0}^{\circ} C_{0}^{\circ}+\left(1-\alpha_{\mathrm{IN}}\right)\left[\frac{D_{\mathrm{w}}^{+}}{E_{\mathrm{IN}}^{+}} C_{\mathrm{w}}^{+}+\frac{D_{\mathrm{w}}^{\circ}}{E_{\mathrm{IN}}^{\circ}} C_{\mathrm{w}}^{\circ}+\frac{D_{\mathrm{w}}^{-}}{E_{\mathrm{IN}}^{-}} C_{\mathrm{w}}^{-}\right]
$$

in which the penetration of non-ionized species into the cytoplasmic bodies and nucleus, all being included in the volume fraction, $\alpha_{\mathrm{IN}}$, and the modification of the intrinsic diffusivity of all drug species by protein adsorption in the aqueous phase within the cell are taken into general account.

After Crank (1956) the solution to equation (24) is

$$
\begin{aligned}
Y_{\mathrm{IN}}=-\frac{2 D_{\mathrm{e}, \mathrm{IN}}}{r b} \sum_{n=1}^{\infty}(-1)^{n} \exp \left(\frac{-D_{\mathrm{e}, \mathrm{IN}} n^{2} \pi^{2} t}{b^{2}}\right) \\
\cdot n \pi \sin \frac{n \pi r}{b} \int_{0}^{t} \exp \left(\frac{D_{\mathrm{e}, \mathrm{IN}} n^{2} \pi^{2} \lambda}{b^{2}}\right) \cdot \phi(\lambda) \mathrm{d} \lambda .
\end{aligned}
$$

The concentration $Y_{\text {IN }}$ at $r=0$ is the limit of equation (26) as $r \rightarrow 0$. Thus,

$$
\begin{aligned}
Y_{\mathrm{IN}}=-\frac{2 D_{\mathrm{e}, \mathrm{IN}}}{b^{2}} \sum_{n=1}^{\infty}(-1)^{n} \exp \left(\frac{-D_{\mathrm{e}, \mathrm{NN}} n^{2} \pi^{2} t}{b^{2}}\right) \\
\cdot(n \pi)^{2} \int_{0}^{t} \exp \left(\frac{D_{c, \mathbb{N}} n^{2} \pi^{2} \lambda}{b^{2}}\right) \cdot \phi(\lambda) \mathrm{d} \lambda .
\end{aligned}
$$

The surface concentration at $r=b$ varies with time by the function $\phi(\mathrm{t})$. Once $\phi(t)$ is found the total rate of drug transport can be readily solved by numerical computation of the steady-state rate in the membrane by equation (22) and the non-steady-state rate in the cell interior by (26). We note 
that the variable surface concentration just inside the cell interior is related to the concentration in the membrane by the effective partition coefficient, $K_{\mathrm{e}, b}$, in equation (18). Nevertheless, $\phi(\mathrm{t})$ is not generally known.

The physical role of the heterophase cell interior that bears upon the movement of drug species needs some explanation (Higuchi \& Higuchi, 1960; Barrer, 1968; Hills, 1968). It is impossible to give an exact description of the influence of the dispersed phase (endoplasmic reticulum, ribosomes, mitochondria, nucleus, etc.) in this model as well as model 3 to follow due to: (a) geometric complexity arising from microirregularities of surfaces; (b) lack of knowledge of microscopic shapes, e.g. spheres, cylinders, ellipsoids, irregular shapes, etc., with respect to their concentration and concentration distribution, size and size distribution and orientation; (c) lack of knowledge of the composition and permeabilities of cellular organelles comprising the dispersed system; (d) complexity arising from the rigidity or elasticity and motility of the dispersed bodies. Cognizant of the difficulties in formulating exact expressions that will be experimentally impossible to evaluate, we have disregarded much of the physical details by simply expressing the cytoplasmic bodies and nucleus as $\left(\alpha_{\mathrm{IN}}\right)$ fraction of the cell interior. Therefore, the $D_{\mathrm{e}, \mathrm{IN}}$ in equation (25) expresses the effective diffusivity of the heterogeneous cell interior in which the cytoplasmic matrix is taken as a hypothetical aqueous compartment in parallel with the cell bodies and nucleus as a hypothetical lipoidal compartment.

Thus far, the diffusional transport of drugs into cells has been described by expressions that can be solved without particular difficulty by conventional numerical methods. However, the problem lies in the assignment of proper values to the various constants. Further, the equations are not explicitly helpful in the immediate treatment and interpretation of experimental data. Therefore, we seek an approximate, but analytic, expression to replace equations (22) and (24), which will be useful in experimental situations.

Hence, the decrease in the bulk aqueous concentration of drug is now given by

$$
\log Y_{\mathrm{AQ}}=\log I-\frac{2 A}{2.303 B} t^{1 / 2}+\frac{2 A}{B^{2}} \log \left(1+B t^{1 / 2}\right),
$$

where $I$ is the initial bulk aqueous concentration and the coefficients are

$$
\begin{aligned}
& A=4 \pi N a b P_{\mathrm{e}, \mathrm{M}} / V_{\mathrm{AQ}}, \\
& B=\frac{a P_{\mathrm{e}, \mathrm{M}}}{b K_{\mathrm{e}, a} K_{\mathrm{e}, b}} \sqrt{\frac{2}{D_{\mathrm{e}, \mathrm{IN}}}} .
\end{aligned}
$$

The derivation is described in Appendix A. The $Y_{\mathrm{AQ}}$ follows a non-linear dependent relationship with respect to the square root of time. However, 
at initial rates, $Y_{\mathrm{AQ}}$ follows a first order relationship with time; i.e.

$$
\log Y_{\mathrm{AQ}}=\log I-(A / 2.303) t \text {. }
$$

The $K_{\mathrm{e}, a} K_{\mathrm{e}, b}$ is readily found from the experiment by equation (23) and the permeability coefficient of the plasma membrane from the initial slope by equation (29). In turn, the constant $B$ and, consequently, $D_{\mathrm{e}, \mathrm{IN}}$ can be extracted by non-linear regression analysis of equation (28). Also, if one had information available on the membrane and cell interior with respect to $\mathrm{pH}$, volume fraction of lipids and cytoplasmic bodies and adsorption, a further insight into the behavior of the membrane and cell interior to drug transport can be obtained, providing the model is the correct one.

\section{Rate of Drug Transport into Cells using Model 2}

Here, as soon as the drug permeates through the plasma membrane, the distribution of drug in the composite cell interior is instantaneously established. In other words, the permeability of the membrane is the rate-determining factor for transport.

Accordingly, $Y_{\mathrm{IN}}$ replaces $Y_{\mathrm{IN}, b}$ and equation (22) clearly becomes

$$
\frac{\mathrm{d} Y_{\mathrm{AQ}}}{\mathrm{d} t}=-\frac{4 \pi N a b P_{\mathrm{e}, \mathrm{M}}}{V_{\mathrm{AQ}}}\left(Y_{\mathrm{AQ}}-\frac{Y_{\mathrm{IN}}}{K_{\mathrm{e}, a} K_{\mathrm{e}, b}}\right) .
$$

From Appendix B, the approximate solution is

where

$$
\log Y_{\mathrm{AQ}}=\log I-\left(1 / B_{2}\right) \log \left(1+A_{2} B_{2} t\right)
$$

$$
\begin{aligned}
A_{2} & =4 \pi N a b P_{\mathrm{e}, \mathrm{M}} / V_{\mathrm{AQ}}, \\
B_{2} & =V_{\mathrm{AQ}} / N V_{\mathrm{IN}} K_{\mathrm{e}, a} K_{\mathrm{e}, b},
\end{aligned}
$$

where $V_{\mathrm{IN}}$ is the interior cell volume and the other terms have been previously defined. While the constant $B_{2}$ is directly obtainable from the experimental data, the permeability coefficient can be calculated from the initial slope of the equation,

$$
\log Y_{\mathrm{AQ}}=\log I-\left(A_{2} / 2.303\right) t .
$$

As the permeability of the membrane is expected to be independent of any physical model, it is also consistent that we find that the initial slope of $A_{2}$ of model 2 is identical to that of $A$ from model 1 .

An alternative approach to the evaluation of this model is one that utilizes the equilibrium data of the experiment. Thus,

$$
\log \frac{Y_{\mathrm{AQ}}-Y_{\mathrm{AQ}(\mathrm{eq})}}{I-Y_{\mathrm{AQ}(\mathrm{eq})}}=-\frac{\beta}{2.303} t
$$


where $Y_{\mathrm{AQ}(\mathrm{eq})}$ is the bulk aqueous concentration at equilibrium and

$$
\beta=\frac{4 \pi N a b P_{\mathrm{e}, \mathrm{M}}}{V_{\mathrm{AQ}}}\left(1+\frac{V_{\mathrm{AQ}}}{N V_{\mathrm{IN}} K_{\mathrm{e}, a} K_{\mathrm{e}, b}}\right) .
$$

\section{Rate of Drug Transport into Cells using Model 3}

In this model the rate-determining barriers to equilibrium drug distribution in the monodisperse cell suspension system are postulated to be the plasma membrane and the membranes of $N^{\prime}$ number of cytoplasmic bodies within the cell. The drug distributes instantaneously in the aqueous interior but permeates slowly into the cytoplasmic bodies including the nucleus.

Equation (30) is also applicable in this case. The mass balance of drug in the cell at any time is expressed by

$$
Y_{\mathrm{IN}}=\left(1-\alpha_{\mathrm{IN}}\right)\left(Y_{\mathrm{IN}, \mathrm{AQ}}+Y_{\mathrm{IN}, \mathrm{AQ}}^{*}\right)+\alpha_{\mathrm{IN}} Y_{\mathrm{CYT}} \text {, }
$$

where $Y_{\mathrm{IN}, \mathrm{AQ}}$ and $Y_{\mathrm{NN}, \mathrm{AQ}}^{*}$ are the time-dependent concentrations of free and protein-adsorbed drug, respectively, in the aqueous cytoplasmic matrix and $Y_{\text {CYT }}$ is the concentration of drug in the cytoplasmic bodies of volume fraction $\alpha_{\mathbb{I N}}$. From Appendix $C$ the quasi-stcady-state solution of equation (30) is

in which

$$
Y_{\mathrm{AQ}}=Y_{\mathrm{AQ}(\mathrm{eq})}+\left(I-\gamma-Y_{\mathrm{AQ}(\mathrm{eq})}\right) \mathrm{e}^{-A_{2} t}+\gamma \mathrm{e}^{-\beta_{2} t}
$$

$$
\begin{gathered}
\beta_{2}=\frac{N^{\prime} S P_{\mathrm{e}, \mathrm{CYT}}}{\left(1-\alpha_{\mathrm{IN}}\right) V_{\mathrm{IN}}}\left(1+\frac{1-\alpha_{\mathrm{IN}}}{\alpha_{\mathrm{IN}} K_{\mathrm{e}, \mathrm{CYT}}}\right), \\
\gamma=A_{2}\left(1-\alpha_{\mathrm{IN}}\right) K_{\mathrm{ad}} Y_{\mathrm{AQ}, \mathrm{IN}(\mathrm{eq})} /\left(A_{2}-\beta_{2}\right) K_{\mathrm{e}, a} K_{\mathrm{e}, b}
\end{gathered}
$$

and the terms are defined in Appendix C. When $\left(Y_{\mathrm{AQ}}-Y_{\mathrm{AQ}(\mathrm{eq})}\right)$ is plotted semi-logarithmically against time, a bi-exponential curve results from which the rate and pre-exponential constants can be found.

\section{Conclusion}

Three physical models for the diffusion transport of drugs across membranes of viable mammalian cells in suspension have been mathematically described using both rigorous and approximate methods. This paper is not intended solely to be an exercise in mathematics since the present investigators intend to use the models as guidelines to the design of experiments and interpretation of data to obtain quantitative estimates of those physical parameters significant in transport phenomena and to evaluate the applicability of the models. Systematic modifications of the models to include such physical and chemical effects as osmotic pressure, electrical field, biochemical 
reactions and others may become necessary as the evidence accumulates. Experimental studies are presently being conducted on drug transport using Burkitt lymphoma cell culture suspensions.

We would like to acknowledge the electron microscopy work of Dr Booe Il Ma, USPHS Special Fellow (F03-DE-42829-01) of The University of Michigan. This investigation was supported in part by United States Public Health Service grants 1-P13-DE-02731 and GM-13368, National Institutes of Health, Bethesda, Maryland, U.S.A.

\section{REFERENCES}

Bangham, A. D., Standish, M. M. \& Watkins, J. C. (1965). J. molec. Biol. 13, 238.

Barrer, R. M. (1968). In Diffusion in Polymers, chap. 6. (J. Crank \& G. S. Park, eds.) Ncw York: Academic Press.

Bean, R. C., Shepperd, W. C. \& Chan, H. (1968). J. gen. Physiol. 52, 495.

Beckett, A. H. \& Moffat, A. C. (1968). J. Pharm. Pharmac. 20, 239S.

Beckett, A. H. \& Triggs, E. J. (1967). J. Pharm. Pharmac. 19, $31 \mathrm{~S}$.

BikhazI, A. B. (1970). Thesis, The University of Michigan, Ann Arbor.

Christensen, H. N. (1962). Biological Transport. New York: W. A. Benjamin.

CrANk, J. (1956). The Mathematics of Diffusion, p. 87. London: Oxford University Press.

Curran, P. F. \& Schultz, S. G. (1968). In Handbook of Physiology, vol. III, section 6. (C. F. Code \& W. Heidel, eds.) Washington, D.C.: American Physiological Society.

Elfbaum, S. G. \& Laden, K. (1967). J. Soc. cosmet. Chem. 19, 119, 163.

Elfbaum, S. G. \& Laden, K. (1968). J. Soc. cosmet. Chem. 19, 841.

Epstein, M. A., Achong, B. G. \& BarR, Y. M. (1964). Lancet i, 702.

FINKelstern, A. \& CAss, A. (1968). In Biological Interfaces: Flows and Exchanges (Basic Science Symposia of the New York Heart Association), p. 145. Boston: Little \& Brown.

Ghanem, A. H., Hrguchi, W. I. \& Simoneldi, A. P. (1969). J. pharm. Sci. 58, 165.

Ghanem, A. H., Higuch, W. I. \& Simonelli, A. P. (1970). J. pharm. Sci. 59, 659.

Goldberk, A. H. (1968). Thesis, University of Michigan, Ann Arbor.

GoldberG, A. H., Higuchi, W. I., Ho, N. F. H. \& ZoGRAFI, G. (1967). J. pharm. Sci. 56, 1432.

Hrguchr, W. I. \& Higuchr, T. (1960). J. pharm. Sci. 49, 568.

HILLs, B. A. (1968). Bull. math. Biophys. 30, 47.

Kakemi, K., Arita, T. \& Muranishi, S. (1965). Chem. pharm. Bull., Tokyo 13, 861.

Kakemi, K., Sezaki, H., Muranishi, S. \& Tsujimura, Y. (1969). Chem. pharm. Bull., Tokyo 17, 1641 .

LAKSHMinARAYANAIAH, N. (1965). Chem. Rev. 65, 491.

LiAng, K. \& Tong, L. K. J. (1969). J. Phys. Chem. 73, 3125.

LnNG, G. N. (1962). A Physical Theory of the Living State: The Association-Induction Hypothesis. New York: Blaisdell.

MATTOCKs, A. M. (1969). J. pharm. Sci. 58, 595.

Muler, I. R. (1968). In Biological Interfaces: Flows and Exchanges (Basic Science Symposia of the New York Heart Association), p. 209. Boston: Little \& Brown.

PenzotTi, S. C. \& MatTocks, A. M. (1968). J. pharm. Sci. 57, 1192.

Rothblat, G. H., Hartzell, R. W., Mialhe, H. \& Kritchevsky, D. (1966). Biochim. biophys. Acta 116, 133.

Rothri.AT, G. H., Buchko, M. \& KrtTrhevsky, D. (1968). Biochim. biophys. Acta 164, 327.

Scheuplen, R. J., Blank, I. H., Brauner, G. J. \& MacFarlane, D. J. (1969). J. Invest. Derm. 52, 63. 
Sessa, G. \& Weissmann, G. (1968). J. Lipid. Res. 9, 310.

Stern, W. D. (1967). The Movement of Molecules Across Cell Membranes. New York: Academic Press.

Suzukr, A., Higuchi, W. I. \& Ho, N. F. H. (1970). J. pharm. Sci. 59, 651.

Troshin, A. S. (1966). Problems of Cell Permeability. New York: Pergamon Press.

TuwINfr, S. B. (1962). Diffusion and Membrane Technology, chap. 7. New York: Reinhold. WaGNer, J. G. (1968). A. Rev. Pharmacol. 8, 67.

Zwolinski, B. J., Eyring, H. \& Reese, C. E. (1949). J. phys. Chem. 53, 1426.

\section{APPENDIX A}

\section{Linear Approximation of the Rate of Drug Transport into Cells using Model 1}

To begin with, equations (19) to (21) are needed, then

$$
\begin{gathered}
G_{\mathrm{M}}=4 \pi N a b P_{\mathrm{e}, \mathrm{M}}\left(Y_{\mathrm{AQ}}-Y_{\mathrm{IN}, b} / K_{\mathrm{e}, a} K_{\mathrm{e}, b}\right), \\
G_{\mathrm{M}}=-V_{\mathrm{AQ}} \frac{\mathrm{d} Y_{\mathrm{AQ}}}{\mathrm{d} t} .
\end{gathered}
$$

We want to express $Y_{\mathrm{IN}}, b$, the time-dependent drug concentration inside the cell and adjacent to the plasma membrane in terms of $Y_{\mathrm{AO}}$, the bulk aqueous concentration. The continuity of flow through $r=b$ of the membrane is expressed by

$$
G_{\mathrm{M}}=-N D_{\mathrm{e}, \mathbb{N}} 4 \pi b^{2}\left(\frac{\partial Y_{\mathrm{N}}}{\partial r}\right)_{r=b}
$$

and the linear solution is

$$
G_{\mathrm{M}}=N D_{\mathrm{e}, \mathrm{IN}} 4 \pi b^{2} Y_{\mathrm{IN}, b} /(r-b) \quad(r<b),
$$

whereupon its substitution into equation (A1) for $G_{\mathrm{M}}$ gives

$$
Y_{\mathrm{IN}, b}=Y_{\mathrm{AQ}} /\left[\frac{1}{K_{\mathrm{e}, a} K_{\mathrm{e}, b}}+\frac{b D_{\mathrm{e}, \mathrm{IN}}}{a P_{\mathrm{e}, \mathrm{M}}} \cdot \frac{1}{(r-b)}\right] .
$$

Furthermore, on the simplifying assumption that $G_{M}$ per cell has not changed appreciably during the period needed to build up the concentration distribution in the composite interior of the cell, the total amount of drug transported across unit area of membrane at $r=b$ is equal to the amount of drug per unit area of spherical shell of the cell interior. Hence,

$$
\begin{gathered}
\int_{0}^{t} G_{\mathrm{M}}^{\prime} \mathrm{d} t=\int_{b}^{r} Y_{\mathrm{IN}} \mathrm{d} r, \\
G_{\mathrm{M}}^{\prime} t \sim \frac{Y_{\mathrm{IN}, b}}{2}(r-b) \quad(r<b),
\end{gathered}
$$


and with $G_{M}^{\prime}=G_{\mathrm{M}} / N 4 \pi b^{2}$ by equation (A4)

$$
(r-b) \sim \sqrt{2 D_{\mathrm{e}, \mathrm{IN}} t}
$$

The linear concentration-distance distribution is a justified approximation up to a period in which the concentration in the center of the cell is small.

Together with equations (A2), (A5) and (A7) the rate expression of (A1) becomes

$$
\frac{\mathrm{d} Y_{\mathrm{AQ}}}{\mathrm{d} t}=-\frac{4 \pi N a b P_{\mathrm{e}, \mathrm{M}}}{V_{\mathrm{AQ}}}\left[1+\frac{a P_{\mathrm{e}, \mathrm{M}}}{b K_{\mathrm{e}, a} K_{\mathrm{e}, b}} \sqrt{\frac{2 t}{D_{\mathrm{e}, \mathrm{N}}}}\right]^{-1} Y_{\mathrm{AQ}}
$$

and, after integration, we get

$$
\log Y_{\mathrm{AQ}}=\log I-(2 A / 2.303 B) t^{1 / 2}+\left(2 A / B^{2}\right) \log \left(1+B t^{1 / 2}\right),
$$

where $I$ is the initial bulk aqueous concentration and

$$
\begin{aligned}
& A=4 \pi N a b P_{\mathrm{e}, \mathrm{M}} / V_{\mathrm{AQ}} \\
& B=\frac{a P_{\mathrm{e}, \mathrm{M}}}{b K_{\mathrm{e}, a} K_{\mathrm{e}, b}} \sqrt{\frac{2}{D_{\mathrm{e}, \mathrm{IN}}}} .
\end{aligned}
$$

For initial periods, equation (A9) reduces to a first-order expression,

$$
\log Y_{\mathrm{AQ}}=\log I-A t / 2.303 \text {. }
$$

\section{APPENDIX B}

\section{Linear Approximation of the Rate of Drug Transport into Cells using Model 2}

The expression for the flux in the plasma membrane for this model is shown again,

$$
G_{\mathrm{M}}=4 \pi N a b P_{\mathrm{e}, \mathrm{M}}\left(Y_{\mathrm{AQ}}-Y_{\mathrm{IN}} / K_{\mathrm{e}, a} K_{\mathrm{e}, b}\right) .
$$

We assume, as in Appendix A, that the flux has not changed appreciably to build up the drug concentration in the cell. Then the total amount of drug transported through the membrane is

$$
\int_{0}^{t} G_{\mathrm{M}} \mathrm{d} t=-4 \pi N \int_{b}^{0} Y_{\mathrm{IN}} r^{2} \mathrm{~d} r
$$

and, recalling that $G_{\mathrm{M}}=-V_{\mathrm{AQ}} \cdot \mathrm{d} Y_{\mathrm{AQ}} / \mathrm{d} t$,

$$
Y_{\mathrm{IN}}=-\frac{V_{\mathrm{AQ}}}{N V_{\mathrm{IN}}} \cdot t \frac{\mathrm{d} Y_{\mathrm{AQ}}}{\mathrm{d} t},
$$

where $V_{\mathbb{I N}}\left(=\frac{1}{3} \pi b^{3}\right)$ is the volume of the cell interior and the other terms have been previously defined. It follows that the solution to equation (B1) becomes

$$
\log Y_{\mathrm{AQ}}=\log I-\left(1 / B_{2}\right) \log \left(1+A_{2} B_{2} t\right),
$$


where

$$
\begin{aligned}
& A_{2}=4 \pi N a b P_{\mathrm{e}, \mathrm{M}} / V_{\mathrm{AQ}}, \\
& B_{2}=V_{\mathrm{AQ}} / N V_{\mathrm{IN}} K_{\mathrm{e}, a} K_{\mathrm{e}, b} .
\end{aligned}
$$

For initial periods, all but the first term in the expansion of $\log \left(1+A_{2} B_{2} t\right)$ can be neglected, so that

$$
\log Y_{\mathrm{AQ}}=\log I-A_{2} t / 2.303 .
$$

An alternative approach to the evaluation of transport data is as follows. Recognizing that mass balance requires that

$$
-V_{\mathrm{AQ}}\left(\mathrm{d} Y_{\mathrm{AQ}} / \mathrm{d} t\right)=N V_{\mathrm{IN}}\left(\mathrm{d} Y_{\mathrm{IN}} / \mathrm{d} t\right)
$$

at quasi-steady-state and differentiating equation (B1), in which case the combined result is

$$
\frac{\mathrm{d}^{2} Y_{\mathrm{AQ}}}{\mathrm{d} t^{2}}+\frac{4 \pi N a b P_{\mathrm{e}, \mathrm{M}}}{V_{\mathrm{AQ}}}\left(1+\frac{V_{\mathrm{AQ}}}{N V_{\mathrm{IN}} K_{\mathrm{e}, a} K_{\mathrm{e}, b}}\right) \frac{\mathrm{d} Y_{\mathrm{AQ}}}{\mathrm{d} t}=0
$$

the initial and equilibrium boundary conditions resolves the above differential equation. Thus,

$$
\log \frac{Y_{\mathrm{AQ}}-Y_{\mathrm{AQ}(\mathrm{eq})}}{I-Y_{\mathrm{AQ}(\mathrm{eq})}}=-\frac{\beta}{2.303} t
$$

where $Y_{\mathrm{AQ}(\mathrm{eq})}$ is the aqueous equilibrium concentration and

$$
\beta=\frac{4 \pi N a b P_{\mathrm{e}, \mathrm{M}}}{V_{\mathrm{AQ}}}\left(1+\frac{V_{\mathrm{AQ}}}{N V_{\mathrm{IN}} K_{\mathrm{e}, a} K_{\mathrm{e}, b}}\right) .
$$

\section{APPENDIX C}

Linear Approximation of the Rate of Drug Transport into Cells using Model 3

Utilizing the following equation as before in the other models,

$$
\frac{\mathrm{d} Y_{\mathrm{AQ}}}{\mathrm{d} t}=-\frac{4 \pi N a b P_{\mathrm{e}, \mathrm{M}}}{V_{\mathrm{AQ}}}\left(Y_{\mathrm{AQ}}-\frac{Y_{\mathrm{IN}}}{K_{\mathrm{e}, a} K_{\mathrm{e}, b}}\right)
$$

and differentiating with respect to time, we get

$$
\frac{\mathrm{d}^{2} Y_{\mathrm{AQ}}}{\mathrm{d} t^{2}}+A_{2} \frac{\mathrm{d} Y_{\mathrm{AQ}}}{\mathrm{d} t}=\frac{A_{2}}{K_{\mathrm{e}, a} K_{\mathrm{e}, b}} \frac{\mathrm{d} Y_{\mathrm{IN}}}{\mathrm{d} t},
$$

where $A_{2}$ has already been defined in equation (B4). If we are able to express the right side of equation (C2) in terms of some function of time, the solution is simplified. 
The distribution of drug within the aqueous cytoplasmic matrix and cytoplasmic bodies of each cell is accountable by

$$
Y_{\mathrm{IN}}=\left(1-\alpha_{\mathrm{IN}}\right)\left(Y_{\mathrm{AQ}, \mathrm{IN}}+Y_{\mathrm{AQ}, \mathrm{IN}}^{*}\right)+\alpha_{\mathrm{IN}} Y_{\mathrm{CYT}} .
$$

The concentration adsorbed, $Y_{A Q}^{*}, \mathbb{N}$, in the aqueous interior is assumed to be linear with respect to the Langmuir isotherm; thus,

$$
Y_{\mathrm{AQ}, \mathrm{IN}}^{*}=K_{\mathrm{ad}} Y_{\mathrm{AQ}, \mathrm{IN}},
$$

where $K_{\mathrm{ad}}$ is the linear adsorption constant. With equations (C3) and (C4) it follows that

$$
\frac{\mathrm{d} Y_{\mathrm{IN}}}{\mathrm{d} t}=\left(1-\alpha_{\mathrm{IN}}\right)\left(1+K_{\mathrm{ad}}\right) \frac{\mathrm{d} Y_{\mathrm{AQ}, \mathrm{IN}}}{\mathrm{d} t}+\alpha_{\mathrm{IN}} \frac{\mathrm{d} Y_{\mathrm{CYT}}}{\mathrm{d} t} .
$$

The expressions for the rate of transfer of drug from the aqueous interior of each cell into the cytoplasmic bodies are given by

$$
-\left(1-\alpha_{\mathrm{IN}}\right) V_{\mathrm{IN}} \frac{\mathrm{d} Y_{\mathrm{AQ}, \mathrm{IN}}}{\mathrm{d} t}=\sum N_{i}^{\prime} S_{i} P_{\mathrm{e}, \mathrm{CYT}, i}\left(Y_{\mathrm{AQ}, \mathrm{IN}}-\frac{Y_{\mathrm{CYT}, i}}{K_{\mathrm{e}, \mathrm{CYT}, i}}\right)
$$

and

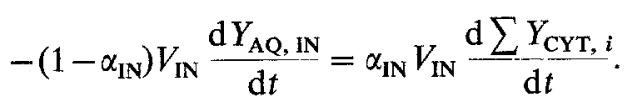

The above equations take into consideration: first, the contribution to the total rate of passive uptake by the $i$ th kind of cytoplasmic bodies (mitochondria, endoplasmic reticulum, etc., including the nucleus) in which there are $N^{\prime}$ number of $i$ th kind bodies with surface area $S_{i}$ possessing a characteristic effective permeability coefficient $P_{\mathrm{e}, \mathrm{CYT}}$ and partition coefficient $K_{\mathrm{e}, \mathrm{CYT}}$; second, the membrane of each kind of body being the rate-controlling barrier. Since it is extremely difficult to experimentally determine the particular physical parameters in equations (C6) and (C7), we proceed to assume an average effect from all the cytoplasmic bodies. Accordingly, after differentiating equation (C6) and then using (C7), the result is

$$
\frac{\mathrm{d}^{2} Y_{\mathrm{AQ}, \mathrm{IN}}}{\mathrm{d} t^{2}}+\beta_{2} \frac{\mathrm{d} Y_{\mathrm{AQ}, \mathrm{IN}}}{\mathrm{d} t}=0
$$

and

$$
\beta_{2}=\frac{N^{\prime} S P_{\mathrm{e} . \mathrm{CYT}}}{\left(1-\alpha_{\mathrm{IN}}\right) V_{\mathrm{IN}}}\left(1+\frac{1-\alpha_{\mathrm{IN}}}{\alpha_{\mathrm{IN}} K_{\mathrm{e}, \mathrm{CYT}}}\right)
$$

whereupon, introducing the boundary conditions of $Y_{\mathrm{AQ}, \mathbb{I N}}(0)=0$ and $Y_{\mathrm{AQ}, \mathrm{NN}}(\infty)=Y_{\mathrm{AQ}, \mathrm{IN}(\mathrm{eq})}$, the solution is

$$
Y_{\mathrm{AQ}, \mathbb{N}}=Y_{\mathrm{AQ}, \mathbb{N}(\mathrm{eq})} \cdot\left(1+\mathrm{e}^{-\boldsymbol{\beta}_{2} t}\right)
$$


and, with equation (C7), the derivative becomes

$$
\frac{\mathrm{d} Y_{\mathrm{AQ}, \mathrm{IN}}}{\mathrm{d} t}=-\frac{\alpha_{\mathrm{IN}}}{\left(1-\alpha_{\mathrm{IN}}\right)} \frac{\mathrm{d} Y_{\mathrm{CYT}}}{\mathrm{d} t}=-Y_{\mathrm{AQ}, \mathrm{IN}(\mathrm{eq})} \cdot \beta_{2} \mathrm{e}^{-\beta_{2} t} .
$$

Finally, by substituting equations (C10) and (C5) for $\mathrm{d} Y_{\mathrm{IN}} / \mathrm{d} t$ of (C2) we have the following second-order, non-homogeneous differential equation, i.e.

$$
\frac{\mathrm{d}^{2} Y_{\mathrm{AQ}}}{\mathrm{d} t^{2}}+A_{2} \frac{\mathrm{d} Y_{\mathrm{AQ}}}{\mathrm{d} t}+\frac{A_{2}}{K_{\mathrm{e}, a} K_{\mathrm{e}, \mathrm{b}}}\left(1-\alpha_{\mathrm{IN}}\right) K_{\mathrm{ad}} Y_{\mathrm{AQ}, \mathrm{IN}(\mathrm{eq})} \beta_{2} \mathrm{e}^{-\beta_{2} t}=0
$$

and letting

$$
\gamma=\frac{A_{2}\left(1-\alpha_{\mathrm{IN}}\right) K_{\mathrm{ad}} Y_{\mathrm{AQ}, \mathrm{IN}(\mathrm{eq})}}{\left(A_{2}-\beta_{2}\right) K_{\mathrm{e}, a} K_{\mathrm{c}, b}}
$$

the integration from initial to equilibrium conditions yields

$$
Y_{\mathrm{AQ}}=Y_{\mathrm{AQ}(\mathrm{eq})}+\left(I-\gamma-Y_{\mathrm{AQ}(\mathrm{eq})}\right) \mathrm{e}^{-A_{2} t}+\gamma \mathrm{c}^{-\beta_{2} t} .
$$

